\title{
The spacing effect in preschool children's free recall of pictures and words
}

\author{
THOMAS C. TOPPINO \\ Villanova University, Villanova, Pennsylvania
}

\begin{abstract}
In two experiments, preschoolers exhibited a spacing effect in free recall of both pictures and words even though procedures were very similar to those used in previous experiments in which preschoolers had not manifested a spacing effect. With these findings, the weight of the available evidence in the literature strongly supports the conclusion that preschoolers generally exhibit a spacing effect in free recall.
\end{abstract}

Repeated exposure to information improves memory for that information. However, the degree of improvement depends upon the spacing between repetitions. When repetitions occur in immediate succession (massed presentations [MPs]), their beneficial effect is often minimal. However, memory improves when repetitions are separated by the passage of time and/or by the occurrence of other events (spaced or distributed presentations [DPs]).

The theoretical mechanisms proposed to account for the effect of spacing repetitions tend to fall into one of two categories. One kind of theory attributes the spacing effect to encoding variability mechanisms (e.g., Bower, 1972; Glenberg, 1977; McFarland, Rhodes, \& Frey, 1979). According to these theories, repetition is beneficial to the extent that information is encoded differently on each occurrence. Spacing benefits performance because, as the spacing between repetitions increases, the degree to which the repetitions are encoded differently is assumed to increase accordingly. According to the other major type of theory, deficient-processing theory, repetition benefits memory to the extent that it increases the total amount or quality of processing devoted to the repeated information. Poor memory of massed repetitions is attributed to deficient processing of one presentation (e.g., Jacoby, 1978; Shaughnessy, Zimmerman, \& Underwood, 1972). As the spacing between repetitions increases, the degree to which both presentations are fully processed increases, and performance increases proportionally.

Theories of the spacing effect also can be categorized according to whether or not the underlying mechanisms are assumed to be under voluntary control. According to voluntary-processing hypotheses, the spacing effect is due to the use of sophisticated mnemonic strategies. Among encoding variability theories, for example, the spacing ef-

\footnotetext{
This research was supported by Grant HD21209 from the National Institute of Child Health and Human Development. I thank Rae Force and Mary Harvey for assisting with the preparation of materials, data collection, and/or data analyses. Correspondence should be addressed to T. C. Toppino, Department of Psychology, Villanova University, Villanova, PA 19085.
}

fect has been linked to the use of organizational strategies (Glenberg, 1977). The successive occurrences of massed repetitions are hypothesized to be organized into the same subjective unit, whereas occurrences of spaced repetitions are hypothesized to be encoded into different subjective units, thereby increasing the probability of retrieval. According to deficient-processing theories based on voluntary processes, subjects choose not to fully process (or rehearse) the second occurrence of massed repetitions (Shaughnessy et al., 1972), perhaps because the information is mistakenly judged to be well learned already (Zechmeister \& Shaughnessy, 1980).

According to involuntary-processing accounts of the spacing effect, the phenomenon reflects the operation of automatically activated processes that may be so fundamental that they are hard-wired into the memory system (e.g., Jensen \& Freund, 1981). For example, it has been proposed that people automatically encode contextual information that is thought to be particularly important for retrieval in free recall (Glenberg, 1979; Greene, 1989). From an encoding variability perspective, retrieval in free recall should be better for spaced-repetition items than for massed-repetition items because the former will have been more likely than the latter to have been encoded with different contextual information on each occurrence. Among deficient-processing theories emphasizing involuntary processes, it has been suggested that one presentation interferes with consolidation of the other presentation when repetitions are massed (e.g., Landauer, 1969; Peterson, 1966) or that the first presentation habituates some aspect of the system so that the subsequent presentations cannot be properly encoded until sufficient time has elapsed (Hintzman, 1974).

Greene (1989) recently presented evidence that both voluntary and involuntary processes may be involved in producing the spacing effect with the type of process depending upon the nature of the task. In a series of studies employing "cued-memory" tasks such as recognition, frequency judgments, and frequency discrimination, Greene obtained a spacing effect with intentional learning procedures, but not with incidental learning procedures. In free recall, however, both intentional and incidental learning 
conditions produced spacing effects, and the effects were of equal magnitude. Greene concluded that voluntary, strategic processes underlie the spacing effect in cuedmemory tasks, whereas involuntary, automatic processes underlie the spacing effect in free recall.

Greene addressed the issue of voluntary versus involuntary processes by investigating the effect of spacing under conditions that are (intentional learning) and are not (incidental learning) conducive to the use of voluntary strategies. An alternative approach is to investigate the spacing effect in populations that vary in the degree to which they employ strategic processes. In this regard, several studies have investigated the spacing effect in preschool children who do not spontaneously engage in the sophisticated forms of strategic processing that have been hypothesized to underlie the spacing effect (e.g., Baker-Ward, Ornstein, \& Holden, 1984; Myers \& Perlmutter, 1978). Unfortunately, the evidence has been inconsistent. Toppino and DiGeorge (1984), in two experiments, failed to find a spacing effect in 4-year-old preschoolers, whereas Rea and Modigliani (1987, Experiment 1) and Toppino (1991, Experiments 1 and 2) obtained a spacing effect in preschoolers of the same age.

The cause of the discrepant findings with preschoolers is unclear. Differences in subject sampling cannot be ruled out as a contributing factor, but it seems unlikely that there was a systematic bias such that Toppino and DiGeorge's 4-year-old subjects were cognitively less advanced than the subjects in the other studies. In particular, Toppino (1991) and Toppino and DiGeorge (1984) sampled subjects from preschools and daycare centers in the same suburban communities, and Toppino (1991) included a sample of 3-year-olds who exhibited a pronounced spacing effect.

Although there were methodological differences among experiments, it is not clear why the existing differences would have produced different results. For example, the methods of Toppino (1991) were very similar to those of Toppino and DiGeorge (1984) with the following exceptions. Toppino's (1991) stimulus pictures (like those of Rea and Modigliani) were taken from a standardized set of black line drawings and were presented on a TV monitor with timing precisely controlled, whereas Toppino and DiGeorge (1984) used full-color pictures taken from children's picture collections and presented them manually with timing only approximately controlled. These differences might be expected to produce more variable data in the less precisely controlled experiments of Toppino and DiGeorge, but there is no obvious reason why these differences would influence the trend relating mean recall performance on massed and spaced repetitions.

The present experiments examined the effect of spacing repetitions on the free-recall performance of preschool children. The primary purpose was to reassess the spacing effect using essentially the same methodology as was used by Toppino and DiGeorge (1984). In addition, the present experiments used both pictures and words as stimuli. Although most experiments in the adult literature on the spacing effect have investigated memory for word lists, only one previous study involving preschool children (Rea \& Modigliani, 1987) has used words as stimuli.

\section{EXPERIMENT 1}

\section{Method}

Subjects and Design. The experiment was designed as a $2 \times 3$ (stimulus type $\times$ repetition/spacing) mixed factorial, with the last factor manipulated within subjects. Depending on stimulus type, either words or pictures were used as stimuli. The repetition/spacing factor consisted of once-presented items (1P) and twice-presented items receiving either massed presentations (MP) or distributed presentations (DP). The subjects were 30 children from suburban nursery schools (mean age $=\mathbf{5 3 . 8}$ months; range $=41$ to 62 months). Eighteen children were assigned to the word condition, and 12 were assigned to the picture condition.

Materials. Six 27-position lists were developed, three using each of two different list structures constructed within the same set of constraints. To control for serial position effects, the first three and the last four positions in each list structure were reserved for $1 \mathrm{P}$ items serving as primacy and recency buffers, respectively. The middle portion of the structures consisted of 20 positions that were designated for the presentation of four 1P items and two presentations of each of four MP items (repetitions separated by no other items) and four DP items (repetitions separated by three item presentations). As a further control on possible extended recency effects (e.g., Underwood, 1969), items were placed in the middle 20 positions such that MP and DP items were equated with respect to the mean serial position of their second occurrences which, in turn, was equated with the mean serial position of $1 \mathrm{P}$ items.

Picture stimuli consisted of color photographs of each of 19 full-color pictures of unrelated common objects. Word stimuli consisted of the dominant label corresponding to each of the picture stimuli.

To construct the first list involving each list structure, pictures were assigned randomly to serve as primacy-buffer, recency-buffer, 1P, MP, and DP items, and then items were assigned to appropriate positions in the list structures. Assignments were made independently for the two structures. A counterbalancing principle was used to generate the second and third lists involving each structure such that, across the three lists, the same items served equally often in each of the three repetition/spacing conditions.

Procedure. Children participated individually. Within each stimulustype condition, an equal number of children received each of the six lists. The experimenter presented the pictures (picture-stimulus condition) or their corresponding labels (word-stimulus condition) at a rate of one item every $5 \mathrm{sec}$. The experimenter presented pictures manually and labels orally. As each picture (or word) was presented, the children labeled (or repeated) it aloud. Following list presentation, the children were allowed $3 \mathrm{~min}$ for oral free recall.

\section{Results and Discussion}

The mean percentages of correct free recall as a function of stimulus type and repetition/spacing condition are presented in the first two rows of Table 1 . The repetition/ spacing factor is actually a combination of two logically separable variables: repetition (once presented vs. twice presented) and the spacing between repetitions (MP vs. DP). The effects of these two variables were examined in separate $2 \times 2$ (stimulus type $\times$ repetition or spacing) analyses of variance (ANOVAs) with repeated measures on the second factor.

The first analysis involving repetition indicated that twice-presented items were recalled better than were 1P items $\left[F(1,28)=14.51, M S_{\mathrm{e}}=355.36, p<.001\right]$, but neither type of stimulus nor the interaction of repetition and type of stimulus approached significance (both $F \mathrm{~s}<$ 
Table 1

Mean Percentages of Correct Free Recall as a Function of Stimulus Type and Repetition/Spacing

\begin{tabular}{llll}
\hline \multirow{2}{*}{$\begin{array}{c}\text { Stimulus } \\
\text { Type }\end{array}$} & \multicolumn{3}{c}{ Repetition/Spacing } \\
\cline { 2 - 4 } & $1 \mathrm{P}$ & MP & DP \\
\hline \multicolumn{4}{c}{ Experiment 1} \\
Pictures & 14.6 & 25.0 & 43.8 \\
Words & 16.7 & 27.8 & 41.7 \\
& Experiment 2 & \\
Pictures & 16.7 & 31.3 & 43.8 \\
Words & 12.5 & 28.6 & 42.7 \\
\hline
\end{tabular}

Note-1P = once-presented items; MP = twice-presented items receiving massed presentations; DP $=$ twice-presented items receiving distributed presentations.

1.00). The second analysis indicated a clear spacing effect with DP items recalled better than MP items $\left[F(1,28)=8.05, M S_{\mathrm{e}}=476.50, p<.01\right]$. Again, neither the effect of type of stimulus nor the interaction of spacing and type of stimulus approached significance (both $F$ s $<1.00$ ).

\section{EXPERIMENT 2}

\section{Method}

Subjects and Design. The design was the same as that of Experiment 1 . The subjects were 48 preschool children (mean age $=53.9$ months; range $=47$ to 59 months). Twenty-four subjects were assigned to the picture-stimulus and word-stimulus conditions, respectively.

Materials and Procedure. Materials and procedure were the same as those of Experiment 1.

\section{Results and Discussion}

The mean percentages of correct free recall as a function of type of stimulus and repetition/spacing are presented in the bottom two rows of Table 1. Again, two separate $2 \times 2$ mixed ANOVAs were conducted. The first analysis indicated that twice-presented items were recalled better than were $1 \mathrm{P}$ items $\left[F(1,46)=37.81, M S_{\mathrm{e}}=\right.$ $282.42, p<.001$ ], but neither the main effect of stimulus type nor the repetition $\times$ stimulus type interaction approached significance (both $F_{\mathrm{s}} \leq 1.11$ ). The second analysis indicated a strong spacing effect with DP items recalled significantly better than MP items $[F(1,46)=$ 22.71, $\left.M S_{\mathrm{e}}=417.99, p<.001\right]$. However, again, neither stimulus type nor the interaction of spacing and stimulus type approached significance (both $F \mathrm{~s}<1.10$ ).

\section{GENERAL DISCUSSION}

In both of the present experiments, 4-year-old preschool children exhibited a spacing effect in free recall of both pictures and words. This replicates the findings of Toppino (1991), who obtained a spacing effect in preschoolers' free recall of pictures, and the findings of Rea and Modigliani (1987), who obtained spacing effects in preschoolers' free recall of both pictures and words.

The present findings were obtained even though procedures were virtually identical to those used by Toppino and DiGeorge (1984) in the only published study to obtain no spacing effect in young children. Why
Toppino and DiGeorge's subjects did not exhibit a spacing effect remains unknown. However, it is now clear that the weight of the available evidence strongly supports the following conclusion: Preschool children typically exhibit a spacing effect in free recall under a variety of experimental conditions.

The fact that preschool children exhibit a spacing effect is most consistent with the hypothesis that the spacing effect is produced by fundamental memory mechanisms that operate automatically (involuntarily). Preschoolers do not ordinarily employ the kind of sophisticated voluntary strategies that have been hypothesized to underlie the spacing effect (see Toppino, 1991, for a brief review). Furthermore, Toppino (1991) found that children as young as 3 years old exhibit a spacing effect in free recall even when to-be-remembered items are presented at a rate that is sufficiently rapid to tax an adult's ability to employ voluntary strategies. Finally, neither Rea and Modigliani (1987) nor Toppino (1991) obtained evidence of developmental changes in the spacing effect in free recall when preschoolers' performance was compared with that of elementary-school children. That is, the size of the spacing effect remained constant during a developmental period marked by increasing strategic sophistication.

Of course, one cannot completely rule out the possibility that some voluntary process, which has yet to be identified, contributes to the spacing effect in free recall. However, it seems that any such process would have to be a very elementary process that is fully mastered by the time children are 3 years old.

\section{REFERENCES}

Baker-Ward, L., Ornstein, P. A., \& Holden, D. J. (1984). The eXpression of memorization in early childhood. Journal of Experimental Child Psychology, 37, 555-575.

BowER, G. H. (1972). Stimulus-sampling theory of encoding variability. In A. W. Melton \& E. Martin (Eds.), Coding processes in human memory (pp. 85-123). Washington, DC: Winston.

GLENBERG, A. M. (1977). Influences of retrieval processes on the spacing effect of free recall. Journal of Experimental Psychology: Human Learning \& Memory, 3, 282-294.

GLENBERG, A. M. (1979). Component-levels theory of the effects of spacing of repetitions on recall and recognition. Memory \& Cognition, 7, 95-112.

GreENE, R. L. (1989). Spacing effects in memory: Evidence for a twoprocess account. Journal of Experimental Psychology: Learning, Memory, \& Cognition, 15, 371-377.

Hintzman, D. L. (1974). Theoretical implications of the spacing effect. In R. L. Solso (Ed.), Theories in cognitive psychology: The Loyola Symposium (pp. 77-99). Hillsdale, NJ: Erlbaum.

JACOBY, L. L. (1978). On interpreting the effects of repetition: Solving a problem versus remembering a solution. Journal of Verbal Learning \& Verbal Behavior, 17, 649-667.

Jensen, T. D., \& Freund, J. S. (1981). Persistence of the spacing effect in incidental free recall: The effect of external list comparisons and intertask correlations. Bulletin of the Psychonomic Society, 18, 183-186.

Landauer, T. K. (1969). Reinforcement as consolidation. Psychological Review, 76, 82-96.

McFarland, C. E., Rhodes, D. D., \& Frey, T. J. (1979). Semanticfeature variability and the spacing effect. Journal of Verbal Learning \& Verbal Behavior, 18, 163-172.

Myers, N. A., \& Perlmutter, M. (1978). Memory in the years from two to five. In P. A. Ornstein (Ed.), Memory development in children (pp. 191-218). Hillsdale, NJ: Erlbaum.

Peterson, L. R. (1966). Short-term verbal memory and learning. Psychological Review, 73, 193-207.

ReA, C. P., \& Modigliani, V. (1987). The spacing effect in 4- to 9year-old children. Memory \& Cognition, 15, 436-443.

Shaughnessy, J. J., Zimmerman, J., \& UnderwoOd, B. J. (1972). Further evidence on the MP-DP effect in free-recall learning. Journal of Verbal Learning \& Verbal Behavior, 11, 1-12. 
TopPINo, T. C. (1991). The spacing effect in young children's free recall: Support for automatic-process explanations. Memory \& Cognition, 19, 159-167.

Toppino, T. C., \& DiGeorge, W. (1984). The spacing effect in free recall emerges with development. Memory \& Cognition, 12, 118-122.

UNDERWOOD, B. J. (1969). Some correlates of item repetition in freerecall learning. Journal of Verbal Learning \& Verbal Behavior, 8, 83-94.
Zechmeister, E. B., \& Shaughnessy, J. J. (1980). When you know that you know and when you think that you know but you don't. Bulletin of the Psychonomic Society, 15, 41-44.

(Manuscript received July 23, 1992.) 